\title{
(Abstracts of papers presented at the TWO HUNDRED AND NINTH MEETING of the Society held from 11th to 12th November 1988 at UNIVERSITY COLLEGE, LONDON)
}

\section{Neutrality of two alleles of Esterase-5 in Drosophila pseudoobscura: a perturbation- reperturbation test}

\section{Einar Arnason \\ Institute of Biology, University of Iceland, Reykjavik, lceland.}

A perturbation-reperturbation tests selective neutrality of $100 / 100 / 100 / 100 / 100$ and $106 / 100 / 100 / 100 / 100$, the two most common alleles at the highly polymorphic $X$-linked locus Esterase-5 in Drosophila pseudoobscura. A total of 22 replicate populations are set up in cages, 11 populations start at a frequency of $76 \%$ and 11 at $21 \%$ of the 106 allele. Allele frequencies change directionally and decrease in both high and low populations as groups and reach equilibria of respectively $60 \%$ and $14 \%$ after $200-300$ days. The directed changes of allele frequencies are due to natural selection. A hypothesis of balancing selection accounts for the pattern of allele frequency changes and predicts a dynamic equilibrium. A neutral hypothesis equally well accounts for the pattern leaving the Est-5 variants to drift at neutral equilibria. A reperturbation of allele frequencies in each population, creating 22 additional reperturbed populations with the original populations as controls, directly addresses the question of balancing selection or dissipated linkage effects. Allele frequencies do not change directionally among the reperturbed populations as a group. The hypothesis of balancing selection is rejected in favor of the hypothesis of dissipated linkage effects. In conclusion the $100 / 100 / 100 / 100 / 100$ and $106 / 100 / 100 / 100 / 100$ are iso-fitness alleles of Est-5

\section{Molecular studies of bovine anti-testosterone immunoglobulins}

\section{T. Jackson, $*$ D. J. Groves, $\dagger$ B. Morris $\dagger$ and P. G. Sanders* \\ * Molecular Biology Group, Department of Microbiology, and $\uparrow$ AFRC Antibody Development Group, Department of Biochemistry, University of Surrey, Guildford GU2 5XH, U.K.}

We are interested in improving the therapeutic properties of ovine and bovine immunoglobulins to hormones involved in the reproductive cycle.

A mouse-bovine heterohybridoma has been produced which secretes a monoclonal antibody against testosterone. In order to investigate the binding of this anti-steroid immunoglobulin to testosterone we have cloned cDNAs for both the heavy and light chain proteins. Studies to characterise these clones will be presented.

\section{Cloning studies of a methionine high-affinity transport system of Salmonella typhimurium}

\section{N. A. Shaw and P. D. Ayling \\ Department of Applied Biology, University of Hull, Hull HU6 7RX, U.K.}

The high-affinity methionine transport system of Salmonella typhimurium (encoded by the met $D^{+}$ locus, formerly called met $P^{+}$) is believed to be of the shock-sensitive category (Cottam and Ayling, unpublished), and therefore should consist of one periplasmic and at least two inner membrane proteins (Ames, G. F. L., 1986, Ann. Rev. Biochem., $55,379)$. A library of a partial Sau 3 A digest of $S$. typhimurium DNA in the replacement vector, $\lambda 1059$, was obtained from Dr. R. Maurer. A series 
of $\lambda 1059$ met $D^{+}$phages was isolated on minimal agar plus D-methionine by lysogenic complementation of an E. coli metD mutant. One of the clones was shown to have retained the activity of a high-affinity methionine transport system.

The met $D^{+}$insert was subcloned into the high copy number plasmid, pUC8, and four met ${ }^{+}$ isolates were selected in an E. coli metD strain. A clone carrying pUC8 with a 1.4 kilobase insert gave a methionine uptake rate which was $30 \%$ that of an $E$. coli met $D^{+}$strain, and showed slow growth on D-methionine medium. Three other larger $m e t D^{+}$plasmids gave wild-type or increased levels of methionine transport.

Expression studies using in vitro and minicell methods showed that the larger met $D^{+}$plasmids encoded two additional proteins of molecular weight 40 and $34 \mathrm{kDal}$. Neither of these proteins appeared to be a periplasmic binding protein.

\section{Conservation of a human} derived $Y$-specific sequence on the $Y$ chromosome of the great apes

\section{Kalaitsidaki, ${ }^{*}$ K. Kwok, $\dagger$ M. A. Ferguson- Smith $\ddagger$ and J. M. Conner* \\ * Duncan Guthrie Institute of Medical Genetics, Glasgow G3 8SJ, U.K. \\ $\dagger$ AFRC Institute of Animal Physiology and Genetics Research, Cambridge, U.K. \\ $\ddagger$ Department of Pathology, Cambridge CB2 1QP, U.K.}

GM6Y10, a human derived repetitive sequence, specific for the short arm of the $\mathrm{Y}$ chromosome, was shown by Southern blot analysis to hybridise with DNA from male gorilla (Gorilla gorilla), male orangutan (Pongo pygmaeus) and male chimpanzee (Pan troglodytes). To investigate its chromosomal localisation the sequence was utilised in in situ hybridisation studies with metaphase chromosomes obtained from chimpanzee, orangutan and gorilla fibroblasts. GMGY10 was labelled by nick translation with biotin-11-dUTP and hybridisation was carried out according to the method of Garson et al. (Nucleic Acids Res., 15, 4761-4770).

Hybridisation signal was observed on the $Y$ chromosome in all 3 cases. In the orangutan, there was signal only on the short arm of the $Y$, whereas in the gorilla the majority of the signal was observed on the long arm. In the chimpanzee it was not possible to decide whether the signal occurred on the short or the long arm because the Y chromosome is particularly small. The possible significance of these findings is discussed.

\section{Identification of a putative B- chromosome specific sequence from rye (Secale cereale)}

\author{
M. J. Sandery, J. W. Forster and R. N. Jones \\ Genetic and Crop Biotechnology Group, Department \\ of Agricultural Sciences, University College of \\ Wales, Aberystwyth, Dyfed SY23 3DD, U.K.
}

DNA from a rye plant containing $6 \mathrm{~B}$-chromosomes was compared by restriction enzyme analysis with DNA from a plant lacking B-chromosomes. With the restriction enzyme Dra 1 , the $6 \mathrm{~B}$ restriction pattern shows the presence of a highly repeated DNA sequence, about $1200 \mathrm{bp}$ in length, which is not visible in the $\mathrm{OB}$ restriction pattern. This repeated sequence has been isolated and used as a probe to investigate whether the sequence is B-chromosome specific, its relation to other rye repeated sequences and its position in the rye $B$ chromosome.

\section{Detection of $\mathrm{Ig}$ and $\mathrm{TcR}$ gene rearrangement in human lymphomas}

\section{Orphanos*, D. Anagnostou, $\dagger$ Th. Papadaki, $\dagger$ G. M. Maniatis* and A. Athanassiadou* \\ * Department of Biology, Medical School, University of Patras, Patras, Greece. \\ $\dagger$ Laboratory of Hematopathology, General Hospital of Athens, "EVANGELIMOS", Athens, Greece.}

$\mathrm{Ig}$ and $\mathrm{T}$ cell receptor gene arrangements represent markers of lineage, clonality and differentiation of $B$ and $T$ cells respectively. Detection of such rearrangements in $B$ and $T$ cell neoplasms has provided the basis for their molecular diagnosis and classification particularly in severe cases where immunological analysis seems to be unable to define lineage and clonality. We used probes which detect rearrangements of the $\operatorname{Ig}$ heavy $\left(J_{H}\right)$ and k-light $\left(C_{K}\right)$ chain genes or the TcR $\beta$-chain $\left(C_{\beta}\right)$ and $y$-chain $\left(T_{y}\right)$ genes and proceeded to genotypic analysis of various cases of lymphoproliferative disorders.

Our results on CLL, Hodgkin's disease and non-Hodgkin's lymphomas agree with those reported in the literature (O'Connor, N. I. J., 1987, J. Pathology, 151, 185-190).

Reactive processes and lymphomas of double phenotype is our main interest. Among the reactive processes we detect cases of $T$ cell lymphoma 
$\left(\mathrm{TcR}_{\beta}\right.$ gene rearranged-Ig genes in germline configuration) as well as of B cell lymphomas ( $\mathrm{Ig}$ genes rearranged-TcR $R_{\beta}$ gene in germline configuration or deleted). One of the former cases was confirmed a year later as a $\mathrm{T}$ cell lymphoma by immunocytochemical analysis. We are currently using a TcR (y) probe in the analysis of reactive processes in order to obtain data concerning the differentiation of these processes.

Ig and $T c R_{\beta}$ genes were rearranged while $T c R y$ gene was in germline in the three cases of lymphomas of double phenotype (B cell lymphomas) studied so far. The evaluation of the usefulness of $\mathrm{TcR}_{\mathrm{y}}$ gene rearrangements in the genotypic analysis of these lymphomas is also desired. Analysis of further cases is in progress.

\section{Selection in karyotypic hybrid zones}

\section{J. B. Searle}

Department of Zoology, University of Oxford, South Parks Road, Oxford OX1 3PS, U.K.

Heterozygotes for chromosomal (structural) rearrangements produced when karyotypic races hybridize in nature are expected to suffer reduced fertility relative to "pure" race individuals, as a result of chromosomal imbalance or loss among germ cells, associated with meiotic aberrations. The width of the zone of hybridisation between the races is expected to vary according to the extent of this fertility reduction. Other selective forces related to karyotype may operate in the hybrid zone. For a certain heterozygous karyotype, selection should favour individuals less prone to meiotic aberrations. There should also be selection for karyotypes which cannot or are unlikely to produce aberration-prone categories of heterozygote.

Studies on the common shrew (Sorex araneus) and house mouse (Mus musculus) indicate that these processes do occur in karyotypic hybrid zones in nature.

\section{Rapid speciation of mycobacteria by infection with DNA transposable elements}

\author{
J. J. McFadden, E. Hull and J. Hermon-Taylor \\ Department of Microbiology, University of Surrey, \\ Guildford, Surrey GU2 5XH, U.K., and Department of \\ Surgery, St. George's Hospital Medical School, \\ London SW17 ORE, U.K.
}

Mycobacterium paratuberculosis (paratb) is the causative agent of Johne's disease (regional enteritis) in ruminants. It is very closely related to Mycobacterium avium with which it shares $98 \%$ DNA homology (McFadden, J. J., 1988, J. Clin. Microbiol., 25, 796-801). However, M. avium causes only sporadic disease in ruminants, whereas paratb is a major pathogen causing chronic, fatal, wasting disease. Conversely, unlike paratb, $M$. avium is a pathogen of birds. Paratb also differs from $M$. avium in its requirement for an ironchelating growth factor (mycobactin) for growth and in its extremely slow growth rate. We have isolated and characterised a mycobacterial insertion sequence (MIS1) which is present in identical multiple copies in the genomes of all strains of paratb, but absent from all strains of $M$. avium. We propose that infection of an $M$. avium strain with MIS1 has recently occurred leading to the rapid evolution of the species $M$. paratuberculosis. We also propose that insertional mutagenesis may account for the multiple phenotypic differences between paratb and $M$. avium. The newly evolved, debilitated paratb may be an example of what Goldschmidt (The Material Basis of Evolution, 1940) termed a "hopeful monster".

\section{Analysis of clines and linkage disequilibrium in Heliconius butterflies}

\section{James Mallet \\ Galton Laboratory, Department of Genetics and Biometry, University College, Wolfson House, 4 Stephenson Way, London NW1 2HE, U.K.}

Narrow zones of hybridisation between colour pattern races are common in unpalatable insect species. Such zones can be maintained if predators attack locally unfamiliar warning colour morphs more readily than locally common morphs: frequency dependent selection would then favour the predominant form on each side of a hybrid zone.

A hybrid zone between races of Heliconius erato was sampled in Amazonian Peru. Three unlinked loci were found to determine the major differences of warning pattern between races. A mark-recapture experiment showed that selection was strong: the fitness of introduced insects with unusual colour patterns was about half that of controls with the normal local colour pattern. Sample data, consisting of gene frequencies and genetic associations, were fitted to theoretical models in order 
to estimate both selection and gene flow indirectly. In Peru, the estimated per locus selection pressures averaged about $20 \%$, and gene flow was about $2 \mathrm{~km}$ per generation.

In this study, direct and indirect measures of selection both showed that selection on individual genes can be strong. In addition, the work illustrates how measurements of hybrid zones can lead to robust inferences about natural selection and population structure in the wild.

\section{Phenotypic plasticity for life history traits in Drosophila melanogaster}

\section{Martin D. Gebhardt and Stephen C. Stearns \\ Zoologisches Institut der Universität Basel, $\mathrm{CH}-4051$ Basel, Rheinsprung 9, Switzerland.}

Developmental time and dry weight at eclosion were analysed genetically in environments different in yeast concentration using a $6 \times 6$ diallel design of crosses. Genetic variances and covariances were strongly influenced by the environment and were different for the two sexes. Strong genetic maternal effects were expressed in the poorer environments. Significant dominance components and a standard test for epistasis suggest that genetic variances were mostly due to allelic interactions. However, computer simulations using an explicit genetic model indicate that this test may be sensitive to small biases of the allelic frequencies due to sampling error.

\section{The effect of food deficiency on the expression of genetic and environmental variances of body size in great tits}

\section{Sabine Henrich and Arie van Noordwijk \\ Zoologisches Institut der Universität Basel, $\mathrm{CH}-4051$ Basel, Rheinsprung 9, Switzerland.}

Heritabilities of final weight and tarsus length of nestlings were measured in a single population during the last three years. Cross-fostering experiments were performed to separate genetic and environmental factors. In contrast to earlier years, food became scarce during the second half of the 1988 breeding season. This influenced final weight and survivorship of nestlings profoundly. The effects of severe food limitation and parent- offspring resemblance and the expression of genetic and environmental variance estimated by full-sib analyses are discussed.

\section{A novel source of genetic variation in ryegrasses}

\author{
R. N. Jones, H. A. Francis and Lutful Hassan \\ Genetics and Crop Biotechnology Group, \\ Department of Agricultural Sciences, University \\ College of Wales, Aberystwyth, Dyfed SY23 3DD, \\ U.K.
}

Colchinine treatment applied to seedlings of perennial ryegrass (Lolium perenne) and Italian ryegrass ( $L$. multiflorum) induces heritable changes in several characters of agronomic usefulness in diploid inbred lines. The changes are stable over several years of vegetative growth in the treatment generation, and they are also transmitted through the selfed-seed to the generation following treatment.

\section{Theoretical and actual causes of gene conversion disparity, for different types of mutation}

\section{B. C. Lamb}

Department of Pure and Applied Biology, Imperial College of Science, Technology and Medicine, London SW7 2BB, U.K.

The possible causes of disparity in the direction of meiotic gene conversion at a heterozygous site are considered, using a wild-type $(+) / \operatorname{mutant}(m)$ example. They include: (i), disparity in the direction of correction of mispairs or non-pairs in hybrid DNA (hDNA); (ii), disparity in the frequencies of invasion by the two types of chromatid ( + bearing and $m$ bearing) in asymmetric hDNA formation; (iii), disparity in the frequencies of invasion by the two strands of a DNA double helix at the site $\left(3^{\prime} 5^{\prime}\right.$ invasions not equal to $5^{\prime} 3^{\prime}$ invasions); (iv), disparity in doublestrand gap frequencies in the two types of chromatid ( + bearing and $m$ bearing) at the site. The amount of disparity is also influenced by the relative frequencies of symmetric and asymmetric hDNA, and of double-strand gaps, by the correction frequencies at the two mispairs, and by interactions of these various factors.

Some of these causes should give disparity for all types of mutation, including large deletions; 
others will only do so for certain types of mutation. Data from fungi are examined to find which of these causes of disparity actually operate. There is strong evidence for (i), (ii) and (iii) occurring in Ascobolus immersus (Zwolinski and Lamb, 1987, Heredity, 59, 155-156, and unpublished).

\section{Phenotypic models of evolution}

\section{John Maynard Smith \\ School of Biology, University of Sussex, Falmer, Brighton BN1 9QG, U.K.}

Usually, phenotypic evolution is of necessity analysed in the absence of specific genetic information, by optimisation theory, or, if fitnesses are frequency-dependent, by evolutionary game theory. What are the advantages and disadvantages of these methods? What assumptions about genetics must hold if they are to be justified? What kinds of problem cannot be treated in this way?

\section{The evolutionary genetics of quantitative characters}

\author{
William G. Hill \\ Institute of Animal Genetics, University of \\ Edinburgh, West Mains Road, Edinburgh EHg 3JN, \\ U.K.
}

Much of our knowledge of the potential rates and extent of evolution of quantitative characters comes from laboratory experiments. These show that directional selection can change traits to well outside the original distribution, and can do so in a few generations. Analyses show that substantial inputs of variation arise from mutation, and theoretical studies suggest and experiments illustrate that this can enable continued responses. So there is obvious scope for adaptation. Indeed it is harder to explain how populations maintain both a nearly constant mean phenotype and much genetic variability.

A fundamental problem in the analysis of short or long term changes in natural populations is to determine what, if any, selective pressures were acting to produce these responses. Conclusions drawn often depend on which traits are actually recorded and are based on one or very few populations, so drift and selection cannot be disentangled.

\section{The evolution of ageing}

\section{Linda Partridge}

Department of Zoology, University of Edinburgh, West Mains Road, Edinburgh EHg 3JT, U.K.

Ageing is a deleterious character whose evolution needs to be explained. The talk will deal with the major theories of ageing and their empirical basis.

\section{Theory, experiment and reality of molecular evolution}

\section{Manfred Eigen \\ Max-Planck-Institut für biophysikalische Chemie, Göttingen, F.R.G.}

The lecture will deal with new concepts in evolutionary biology such as "sequence space", "quasi species" and "statistical geometry". After a brief introduction of these theoretical concepts, experiments will be described that allow for quantitative tests. The experiments include cloning of mutant distribution of RNA molecules produced de novo by $\mathrm{Q}_{\beta}$ replicase and determining their sequences. Similar in vivo experiments using phage $\mathrm{Q}_{\beta}$ and $E$. coli as a host have been carried out. Subjecting such systems to selection pressure causes them to adapt to new environmental conditions. Several types of automatized evolution machines are described. Applications to viral systems will be discussed.

\section{Could natural selection be responsible for molecular evolution and polymorphism?}

\author{
John H. Gillespie \\ Department of Genetics, University of California, \\ Davis, California, U.S.A.
}

A short review of recent work showing that various patterns in the molecular evolution and polymorphism data are not compatible with current versions of the neutral allele theory will be presented. This will be followed by a description of a class of models of molecular evolution and polymorphism due to the action of natural selection that is in remarkable agreement with much of the molecular data. These models have a mathematical structure that is similar to that of the strictly neutral model, although they differ in the predictions about the distribution of nucleotide substitutions. 
As an example, a model of natural selection in a fluctuating environment will be described. This model will be analysed using the method of strong selection, weak mutation limits.

\section{The evolutionary biology of transposable elements}

\section{Brian Charlesworth \\ Department of Biology, University of Chicago, Chicago, Illinois 60637, U.S.A.}

The dynamics of transposable elements in natural populations will be considered from a theoretical standpoint; and the predictions of the various models compared with data from natural populations of Drosophila. Evidence will be presented that most of this information agrees with the view that transposable elements are parasitic DNA.

\section{Metabolic constraints in evolution}

\section{Richard Beeby and Henrik Kacser \\ Department of Genetics, University of Edinburgh, Edinburgh EH9 3JN, U.K.}

We wish to discuss how the metabolic and enzymatic composition of the modern cell evolved from its primitive ancestor. Assuming that such an ancestor contained few and low-activity protoenzymes with very broad specificities, whose nature was determined by some kind of genetic material, one allows these "genes" to mutate and duplicate and delete randomly. The effects of these changes are reflected in the different rates of metabolic output, of which the enzymes themselves are the most important. Clones with different growth rates arise and this constitutes the natural selection in this early phase. The outcome is a diversification of types of enzyme tending towards monospecificity. Early stochastic events determine and restrict the paths such evolution can take, while the inherent metabolic constraints set limits to the phenotypic possibilities. Starting from the same initial composition of the ancestral cell one can "repeat" the evolution but ends up with different "solutions" (in number and types of enzymes) while the final growth rates are not greatly different. The modern cell is one of these, relatively arbitrary, solutions which thereby determines the nature of the genes which sustain it.

\section{Comparative molecular embryology as an approach to study the relationship between development and evolution}

\author{
Michael Akam,* Alfonso Martinez-Arias, $\dagger$ \\ lain Dawson,* Guy Tear* and Robert Kelsh* \\ * Department of Genetics and † Department of \\ Zoology, Downing Street, Cambridge CB2 3EH, U.K.
}

The homeotic and segmentation genes of Drosophila form a regulatory hierarchy that directs pattern formation in the early embryo. Many of these genes have been highly conserved in evolution, so it is possible to identify their structural homologues in distantly related species. We have embarked on a project to identify these homologues in the locust Schistocerca gregaria. Our object is to relate differences in the morphology and development history of these two insects to changes in the regulation and function of specific genes.

\section{The interconversion of purine ribonucleotides in wild-type and APRT-deficient Friend erythroleukaemia cells}

\section{Franklin M. Amara and P. Gerald McKenna \\ Biomedical Sciences Research Centre, University of Ulster, Coleraine BS52 1SA, Northern Ireland.}

In Friend mouse erythroleukaemia cells, deficiency of the purine salvage pathway enzyme, adenine phosphoribosyl transferase (APRT) results in increased sensitivity to mutagenesis (Wilkinson, Y. A. et al, 1988, Genome, 30, suppl. 1; Ward, P. E. and McKenna, P. G., 1987, Mutation Res., 180, 273-276). Wild-type Friend cells (clone 707) were compared with APRT-deficient mutant subclones (707 DAP8 and 707 DAP10) with respect to the metabolism of labelled exogenous hypoxanthine. The APRT-deficient subclones were found to have higher levels of two enzymes of the de novo purine pathway, adenylosuccinate synthetase (356\%, $481 \%$ ) and guanosine 5 -monophosphate reductase $(178 \%, 161 \%)$ relative to clone 707 . The relative enzyme activities paralleled the enhanced capacity for the incorporation of hypoxanthine to adenine rather than to guanine nucleotides in the ratios of 10 and $\mathbf{1 1 . 9 2}$ for the APRT-deficient subclones relative to wild-type cells. There was also a significantly increased turnover rate of inosine 
5 -monophosphate in the APRT-deficient subclones relative to wild-type cells.

It is proposed on the basis of these results that in Friend cells, the lack of the salvage pathway enzyme APRT may be partially compensated for by the divergent capacity of the hypoxanthine metabolism in the purine de novo pathway to selectively yield adenine nucleotides for ATD-dependent processes.

\section{The role of}

deoxyribonucleotide triphosphate pools in the maintenance of errorfree DNA repair processes in Friend mouse erythroleukaemia cells

\section{Yvonne A. Wilkinson and P. Gerald McKenna \\ Biomedical Sciences Research Centre, University of Ulster, Coleraine BT52 1SA, Northern Ireland}

Friend mouse erythroleukaemia cells deficient in the salvage pathway enzyme, thymidine kinase, exhibit increased sensitivity to cell killing and mutagenesis by: the physical mutagens, ultraviolet irradiation (McKenna, P. G. and Hickey, I., 1981, Cell Biology International Reports, 5, 555-561) and gamma irradiation (McKenna, P. G., McKelvey, V. J. and Frew, T. L., 1988, Mutation Research, $200,231-242)$ and by the chemical mutagens: ethyl methanesulphonate, methyl methanesulphonate, $\quad \mathrm{N}$-methyl- $\mathrm{N}^{\prime}$-nitro- $\mathrm{N}$-nitrosoguanidine and mitomycin C (McKenna, P. G. and Yasseen, A. A., 1982, Genetical Research, 40, 207-212). Deoxyribonucleotide triphosphate pool measurements in cycling Friend erythroleukaemia cells show differences between wildtype clone 707 cells and thymidine kinase-deficient clone 707 BUF cells (dATP-130\%, dTTP-39\%, dGTP-50\%, dCTP-99\%, representing dNTP levels in clone 707 BUF cells as a percentage of the levels in wild-type clone 707 cells). Thus pool differences which already exist in cycling clone 707 BUF cells, together with any dNTP pool changes induced by mutagen treatment may render DNA repair processes error-prone and thus account for the increased mutagen sensitivity observed in thymidine kinase-deficient Friend erythroleukaemia cells.

This research was supported by the Leukaemia Research Fund.
24. Analysis of aprt mutants of the Friend erythroleukaemia cell line

\section{Rosemary O'Neill and Ivor Hickey \\ Department of Biology, The Queen's University of Belfast, Belfast BT7 INN, Northern Ireland.}

Clone 707 of the Friend erythroleukaemia cell line (FEL) has been shown to exhibit high mutation rates for two genes, thymidine kinase and adenine phosphoribosyl transferase, while being stable for other markers including resistance to thioguanine, ouabain and cytochalasin B. Loss of thymidine kinase, as in a number of other rodent cell lines, is associated with DNA methylation. We have examined a large number of independently isolated aprt mutants to determine whether they can be restored to wild-type by treatment with the hypomethylating agent 5 -azacytidine. In all cases it was found that although treated cells could form clones in the selective medium AAT they were unable to utilise exogenous adenine.

The aprt gene is located on mouse chromosome 8. This is easily identified in FEL cells as a centric fusion with chromosome 6 . Each cell contains two copies of this fusion product. The two $6 / 8$ chromosomes may be distinguished as only one can be silver stained. We have cytogenetically examined spontaneous and mutagen induced aprt mutants to determine if loss or balanced reduplication of one copy of this chromosome was responsible for the high rate of mutation. All mutant clones retained both copies of this chromosome indicating that chromosomal changes are not involved in the production of aprt mutants.

\section{An epigenetic theory of rapid evolution}

\section{J. Pritchard}

Department of Human Genetics, University of Newcast/e upon Tyne, Newcastle upon Tyne NE2 4AA, U.K.

Conventional evolutionary theory fails to account adequately for the speed of adaptive evolution, or for apparent differences in evolutionary stability of phenotypic strains. A new theory is proposed that provides an explanation of these features, as well as convergent evolution, canalised development, heterochrony and the coexistence of regulative and mosaic developmental controls. A common evolutionary theme is recognised in the 
progressive attainment of genomic independence of the external world.

All organisms acclimatise to their surroundings, but individuals vary in this capacity. It would be expected that alleles which favour acclimatisation and which are already dispersed throughout a population would accumulate in survivors of continued environmental selection. Experiment has shown that under such conditions alreadyadapted individuals can eventually arise. Evolution of preadapted states involves recognition of internal stimuli which replace those that originally impinged on the organism from outside. This internalisation of control is seen as the first step in the progressive genetic underpinning of phenotypic adaptation to specialised conditions.

With continued selection, late acting stimuli are considered to be replaced by others acting earlier in ontogeny, so that development of adaptive traits becomes initiated at progressively earlier stages (heterochrony), or is reinforced by additional stimuli (canalisation). Eventually the most ancient and fundamental traits could become defined by morphogens laid down in the egg or early embryo, in accordance with mosaic, as distinct from regulative control. Such a sequence should allow phenotypic specialisation to proceed relatively rapidly towards perfect adaptation. 